\title{
PCA in smart growth evaluation
}

\author{
Huanjing Lao \\ North China Electric Power University(Baoding), Baoding 071000, China. \\ 547458477@qq.com
}

Keywords: Principal Component Analysis(PCA),smart growth

\begin{abstract}
We nowadays attach more attentions to the concept of sustainable development. Making our life develop faster, suitable and more economical becomes an urgent problem to solve. In these years, some of the cities' continued urban sprawl and the loss of farmland which is surrounding urban centers causes their development out of balance. It does great harm to a country as well as people's happiness. Smart growth is a theory which comprehensively gives the solution to this problem. We establish a basic metric model based on Principal Component Analysis, selecting Chishui city and Heshan city of China as our research objects to do some comparison. By collecting 16 cities' data from the provinces where the two cities locate, we build our initial evaluation model and it is consist of 4 first class indicators and 20 second class indicators . By using SPSS to analyze these data, 8 principal components are accepted finally. Till now, we work out that the success of smart growth of Chishui is -0.1396 , while Heshan is 0.2238 .
\end{abstract}

\section{Introduction}

\subsection{Backgrounds}

We nowadays attach more attentions to the concept of sustainable development. Making our life develop faster, suitable and more economical becomes an urgent problem to solve. In these years, some of the cities' continued urban sprawl and the loss of farmland which is surrounding urban centers causes their development out of balance. It does great harm to a country as well as people's happiness. Smart growth is a theory which comprehensively gives the solution to this problem.

Firstly, we should take into account that both small towns and rural areas needs setting a goal for a more equitable society, more prosperous economy, and much better environment to develop continuously in the long run. Secondly, with/compare to the rapid urbanization changes/development, the construction of a city seems more important. Not only do we consider the current carrying capacity, but also the re-planning of traffic scope expansion of our cities. Also, population expansion needs paying attention to discussing. Due to the fact that smart growth focuses on building cities, establishing an evaluation model based on this theory seems reasonable and practical.

We will establish a metric to measure the success of smart growth of a city and discuss the current plans.

\subsection{Restatement of the Problems}

In order to achieve sustainable and stable development planning objectives in the long term, we should establish a reasonable model to evaluate the urban development plan and then give our advisable measures. Considering demographics, growth needs, and geographical conditions of a city as well as other important factors, we will proceed as follows:

- Define a metric to measure the success of smart growth of a city.

- Research the current growth plan of the cities selected and measure how the current plan of each city meets the smart growth principles. 


\section{Model}

\subsection{Basic Metric Model-Based on PCA}

\subsubsection{Construction of the Basic Model}

Principal Component Analysis(PCA) is a statistical procedure that use orthogonal transformation to convert a set of observations of possibly correlated variables into a set of values of linearly uncorrelated variables called principal components.

In order to secure the principle that data loss least, it simplifies multivariable section data furthest and use dimensionality reduction to visualize and process high-dimensional datasets. For the evaluation model-smart growth metric model (SGM) which we aim to build, many factors including environment, society, population, traffic and so on, need taking into consideration. Based on the Principal Component Analysis(PCA), we can screen out the principal elements with significant variations between the success of a city's smart growth and the factors mentioned above and then find the relevance. Thus it is suitable for our initial building of the assessment system.

2.1.2 Results and Analysis of the Model

The objective cities we select are Chishui and Heshan. The reason for choosing them is the huge gap between these two cities. And to establish our evaluation model, such factors that affect the smart growth of the city need to be taken into account.

Firstly, We classify all of these different aspects into four categories including population, economy, environment and society. And then based on the characteristics of each part, we select 20

The First level indicator

\begin{tabular}{|c|c|c|}
\hline \multirow{5}{*}{ population } & Natural growth rate of population & $x_{1}$ \\
\hline & Pure mobility ratio & $x_{2}$ \\
\hline & Population Aging Index & $x_{3}$ \\
\hline & Non-agricultural population proportion of the total population & $x_{4}$ \\
\hline & Average education year & $x_{5}$ \\
\hline \multirow{4}{*}{ economy } & local financial revenue & $x_{6}$ \\
\hline & The increase proportion of tertiary industry & $x_{7}$ \\
\hline & unemployment rate & $x_{8}$ \\
\hline & per capita GDP & $x_{9}$ \\
\hline \multirow{5}{*}{ environment } & Per capita public green area & $x_{10}$ \\
\hline & Average annual reduction rate of cultivated land area & $x_{11}$ \\
\hline & So2 emissions per square kilometer & $x_{12}$ \\
\hline & Per capita dust emissions & $x_{13}$ \\
\hline & The proportion of pollution control investment in GDP & $x_{14}$ \\
\hline \multirow{6}{*}{ society } & Average Wages of Staff and Workers & $x_{15}$ \\
\hline & Per capita living space & $x_{16}$ \\
\hline & The number of doctors per 10,000 population own & $x_{17}$ \\
\hline & coverage area of built-up area & $x_{18}$ \\
\hline & The number of criminal cases per 100,000 population & $x_{19}$ \\
\hline & The average annual growth rate of investment in education & $x_{20}$ \\
\hline
\end{tabular}

indicators to do PCA analysis. The specific division has been performed in the table.

From the collected data of large amount, we finally get some useful indicators which mostly influence the development. 


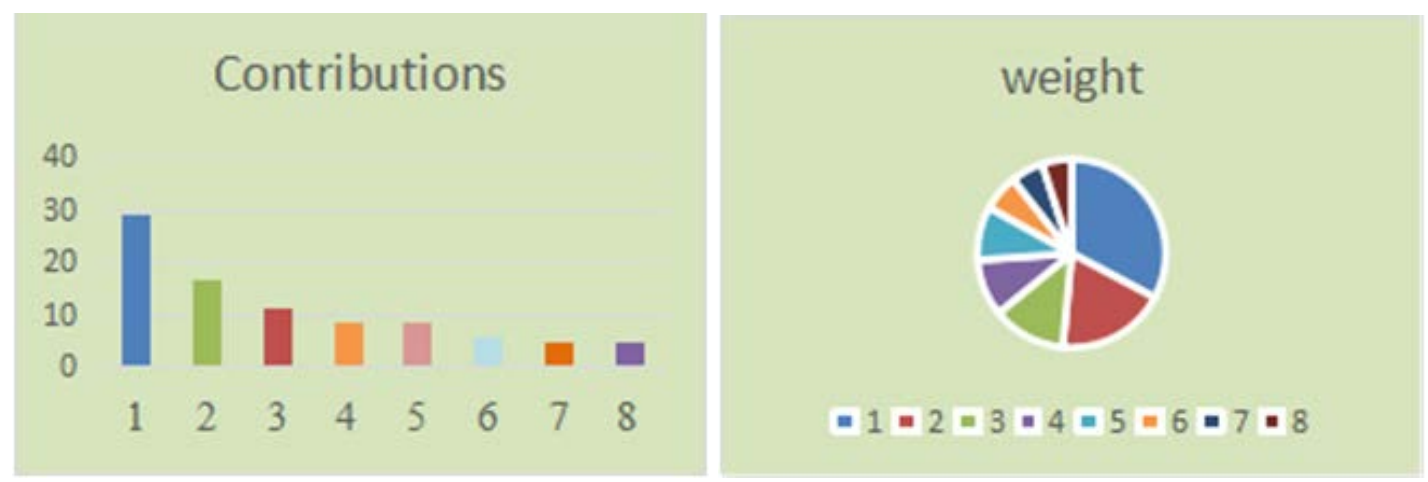

After calculation, We integrated 20 indicators which are considered above into eight principal components, their cumulative contribution rate is over $89 \%$. So the eight main components can together perform the development of various cities clearly and comprehensively. The contribution rates of each principal component are pictured in figure $\mathrm{x}$, and the weights are in figure $\mathrm{y}$. Obviously, the first principal component accounts for a large proportion in every aspect.

Then we obtain the score of each principal component in two cities.

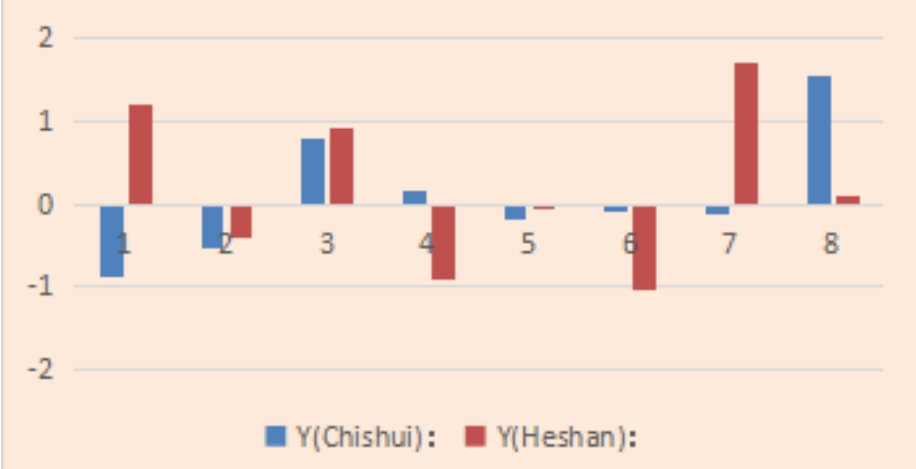

There is a big difference of their scores. We can conspicuously find that Chishui's first principal component score is negative. As we mentioned above, the first principal component accounts for a large proportion in every respect. Thus, Chishui's comprehensive score will be definitely lower than Heshan. And our hypothesis will be verified in the next discussion.

Now, we obtain the comprehensive formula of success scores of smart growth.

$$
\begin{aligned}
& Z=0.3277 y_{1}+0.1873 y_{2}+0.1261 y_{3}+0.0976 y_{4}+0.0925 y_{5}+0.0625 y_{6}+0.0577 y_{7}+0.0505 y_{8} \\
& Z_{\text {Hesha }}=0.2238 ; \\
& Z_{\text {Chishui }}=-0.1396
\end{aligned}
$$

\section{Conclusions}

It's necessary to explain that one of the scores are negative numbers. Since most of the sample cities have a larger population compared to Chishui, some numbers behave negative indicating that the corresponding indicators' levels are below the average. Thus, Chishui's smart growth extent is lower than the average while Heshan seems better.

\section{References}

[1]. wikipedia,Retrieved from https://en.wikipedia.org/wiki/Smart_growth

[2]. Linan Luo1,Lifu Xie 2-.Analysis of climatic resources of Ecotourism in Chishui city.Journal Of Guizhou Meteorology.2010;34(01):0030-03.

[3]. FeiZheng,Zhuying Zhang.Research on the Coordinate Development Models of the Learning City Large-scale Systems.Value Engineering.2016;06:0245-03. 
[4]. Peng Du1, Bing Xia2,Lei Yang3.Study on the Valuation Index System of National Central City Intelligent Development.Science \& Technology Progress and Policy.2016;30(06):0108-05.

[5]. Peiji Shi,Yinfeng Yang,Yanfang Wu. Evaluation Model of Urban Sustainable Development Coordination Based on Compound System.Statics and Decision Making.2010;14:0036-03 